\title{
KOSZUL HOMOLOGY AND THE STRUCTURE OF LOW CODIMENSION COHEN-MACAULAY IDEALS
}

\author{
WOLMER V. VASCONCELOS
}

\begin{abstract}
The relationship between the properties of the Koszul homology modules of two ideals connected by linkage is studied. If the ideal $I$ is either (i) a Cohen-Macaulay ideal of codimension 3, or (ii) a Gorenstein ideal of codimension 4, the one-dimensional Koszul module carries considerable information on the structural nature of the linkage class of $I$ in case (i), or on the conormal module of $I$ in case (ii). Emphasis is given to the verification of the properties by computation.
\end{abstract}

Introduction. The aim of this paper is to examine the structure of Cohen-Macaulay ideals of low (i.e., 3 and 4) codimension as it is expressed by the Koszul homology modules. More specifically, given a perfect ideal $I$ of a Gorenstein ring $R$, we are interested in how (i) its numerical data (e.g., Betti numbers and degrees of its generating syzygies in the case of a homogeneous ideal), (ii) properties of the full linkage class of $I$, and (iii) whether $I$ is suitably generated (e.g. generated by a $d$-sequence) are mirrored on the module and the algebra structures of the homology of the Koszul complex build on a generating set of $I$.

The main result (Theorem 2.4) is the proof that for a perfect ideal $I$ of codimension three, the condition that the 1-dimensional Koszul homology module $H_{1}(I ; R)$ be Cohen-Macaulay is an invariant of the full linkage class of $I$. The framework for this result is provided by several facts. First, the classical descriptions of Cohen-Macaulay ideals of codimension two and of Gorenstein ideals of height three as belonging to the linkage classes of complete intersections $[\mathbf{A}, \mathbf{B E}, \mathbf{G a}, \mathbf{W}]$. Then, a theorem of Huneke $\left[\mathbf{H u}_{\mathbf{1}}\right]$ asserting that the property " $H_{i}(I ; R)$ is CohenMacaulay for $i \leqslant m$ " is an invariant of the even linkage class of $I$. Finally, in case of ideals of polynomial rings, data on the Koszul homology has the advantage of being in principle 'known at the outset,' that is, hypotheses on them can be verified once the ideal has been specified by a set of generators. (In the last section we indicate how symbolic computation packages may be used to obtain this information.) To contrast, already in the case of codimension four Gorenstein ideals the depths of the Koszul homology modules of directly linked ideals may differ sharply. Furthermore, in codimension three, we show that this invariance does not extend to the next Koszul module. To further enhance the role of $H_{1}(I ; R)$ as a carrier of

Received by the editors June 16, 1986.

1980 Mathematics Subject Classification (1985 Revision). Primary 13H10; Secondary 13C05, 13 C15.

Key words and phrases. Linkage, Cohen-Macaulay ideal, Gorenstein ideal, Koszul homology.

Partially supported by the National Science Foundation. 
information on the 'structure' of $I$, we consider diverse ways of connecting the depth of $H_{1}(I ; R)$ to the divisor class of the canonical class of $R / I$.

The module $H_{1}(I ; R)$ plays a different role in the theory of Gorenstein ideals of height four. The relationship between the Cohen-Macaulayness of $H_{1}(I ; R)$ and of the conormal module $I / I^{2}$ (Theorem 3.1), first studied in [VV], is shown here to hold both ways. It was used in [loc. cit.] to extend a result of Herzog-Miller [HM] proving that six-generated Gorenstein ideals are determinantal. The methods also serve to show (Theorem 3.7) that for Gorenstein ideals of codimension four being generated by a $d$-sequence (see $\$ 1$ ) places severe restrictions on the module structure of $I / I^{2}$. In this manner, a question of Huneke [ $\left.\mathbf{H u}_{3}\right]$, first verified on the computer, gets explained.

The third and last problem discussed turns on a conjecture (cf. §4) that in codimension three, the only syzygetic ideals (see $\$ 1$ ) whose conormal modules are Cohen-Macaulay are Gorenstein ideals. The evidence for it comes from the numerical data of a narrow but extensive class of examples, the ideals with pure resolutions. The results of $\$ 2$ are used to obtain constraints on the numerical data of an ideal if its Koszul homology is to be Cohen-Macaulay.

1. Syzygetic ideals and linkage. This preliminary section introduces terminology and some additional background. Throughout $R$ will be a Gorenstein local (resp. graded) ring and $I$ a Cohen-Macaulay (resp. homogeneous Cohen-Macaulay) ideal of height $g$. The quotient $R / I$ will be denoted by $S$. The notation $v(M)$ will denote the minimum number of generators of the $R$-module $M$.

Three notions play ubiquitous roles here: the Koszul homology of the ideal $I$, the linkage class of $I$, and-in the case of a codimension three ideal-the canonical module of $S$. We review them briefly and bring out some of the connections.

(1.1) Koszul homology. Given a generating set $\mathbf{x}=\left\{x_{1}, \ldots, x_{n}\right\}$ of $I$, we denote by $\mathbf{K}$ the Koszul complex built on $\mathbf{x}$. The differential $\partial$ of $\mathbf{K}$ is also a derivation on the exterior algebra structure of $\mathbf{K}$ : if $\omega$ and $\omega^{\prime}$ are homogeneous elements of $\mathbf{K}$ of degrees $p$ and $q$ respectively, then $\partial\left(\omega \wedge \omega^{\prime}\right)=(-1)^{p} \omega \wedge \partial\left(\omega^{\prime}\right)+$ $\partial(\omega) \wedge \omega^{\prime}$. This implies that the module of cycles $\mathbf{Z}=\mathbf{Z}(\mathbf{K})$ is a subalgebra of $\mathbf{K}$ and that the boundaries, $\mathbf{B}$, form a two-sided ideal of $\mathbf{Z}$. Therefore the homology of the Koszul complex, $H_{*}(\mathbf{K})$, inherits a skew-commutative $R$-algebra structure.

It is with the module structure of $H_{*}(\mathbf{K})=\oplus H_{i}(\mathbf{K})$ that we shall be most concerned with. The first module, $H_{0}(\mathbf{K})$, is just $R / I$, while the last nonvanishing module, $H_{n-g}(\mathbf{K})$, is identified to $\operatorname{Ext}^{g}(R / I, R)$, and so these are invariantly defined. The other modules may, however, depend on the chosen set of generators $\mathbf{x}$, but in a completely predictable form. For instance, if $R$ is a local ring and $n$ is picked minimal, then $H_{*}(\mathbf{K})$ is independent of the chosen elements. We simply write $H_{i}(I ; R)$, or $H_{i}(I)$-or even just plain $H_{i}$-for these modules.

(1.1.1) Definition. Two desirable properties of $H_{*}(\mathbf{K})$ are embodied in the following definitions. We assume that $R$ is a local ring.

(i) (SCM). $I$ is strongly Cohen-Macaulay if the modules $H_{i}(I ; R)$ are CohenMacaulay (cf. [Hu $\mathbf{1}]$ ). 
(ii) (SD) $I$ has sliding depth if depth $H_{i}(I ; R) \geqslant \operatorname{dim}(R)-n+i$, for all $i$ (cf. $\left.\left[\mathrm{HSV}_{2}\right]\right)$.

Both properties are independent of the generating sets and localize. They have an important role in deciding when the associated graded rings of $I$ are Cohen-Macaulay or Gorenstein (cf. $\left.\left[\mathbf{H S V}_{\mathbf{2}}\right]\right)$. More often, however, one is likely to find that these conditions only hold for a subrange of $i$, or even scattered values of $i$.

(1.1.2) EXAMPLE (CF. [AH]). Let $I$ be a perfect ideal of codimension two,

$$
0 \rightarrow R^{n-1} \rightarrow R^{n} \rightarrow I \rightarrow 0 .
$$

The module of 1-cycles of $\mathbf{K}, \mathbf{Z}_{1}$, is the submodule $R^{n-1}$ of this resolution. Since $\mathbf{Z}$ is a skew-commutative algebra, the natural mapping $\mathbf{Z}_{1} \times \cdots \times \mathbf{Z}_{1} \rightarrow \mathbf{Z}_{i}$ factors through $\Lambda^{i} \mathbf{Z}_{1} \rightarrow \mathbf{Z}_{i}$. But both $\Lambda^{i} \mathbf{Z}_{1}$ and $\mathbf{Z}_{i}$ are reflexive modules which are naturally isomorphic in codimension one; it follows that they are globally isomorphic. Thus each $\mathbf{Z}_{i}$ is free, and it follows easily that $I$ is strongly Cohen-Macaulay.

(1.1.3) Definition. We now recall a hierarchy of conditions on the ideal $I$ that is closely related to its Koszul homology.

First, there exists a natural exact sequence:

$$
H_{1}(I ; R) \rightarrow S^{n} \rightarrow I / I^{2} \rightarrow 0 .
$$

The kernel of this sequence is denoted by $\delta(I)$. We shall say that $I$ is syzygetic if $\delta(I)=0$. This module can be identified to the second lower deformation module $T_{2}(S / R, S)$, so that it is independent of the set of generators. Some of the higher deformation modules are also expressible in terms of Koszul homology (cf. $\left[\mathbf{H e}_{3}\right]$ ). $\delta(I)$ was described in $[\mathbf{S V}]$ in the more amenable form: Consider the canonical map from $\operatorname{Sym}_{2}(I)$, the symmetric square of $I$, onto $I^{2}$,

$$
\alpha: \operatorname{Sym}_{2}(I) \rightarrow I^{2} \rightarrow 0 .
$$

Then $\delta(I)=\operatorname{ker}(\alpha)$. We indicate shortly how the vanishing of $\delta(I)$ may be ascertained.

The map $\alpha$ is but a component of a more general homomorphism. There is a natural map from the symmetric algebra of $I$ to its Rees algebra:

$$
\psi: \operatorname{Sym}(I) \rightarrow \mathscr{R}(I)=\sum I^{n} t^{n} \subset R[t] .
$$

$I$ is said to be of linear type, if $\psi$ is an isomorphism. (See [ $\left.\mathbf{H S V}_{\mathbf{1}}\right]$ for some of the properties of these ideals.)

To express the kernels of both $\alpha$ and $\psi$ (note that $\alpha$ is the degree 2 component of $\psi$ ) we proceed in the following manner.

Let $I=\left(f_{1}, \ldots, f_{n}\right)$. The natural presentation of $\mathscr{R}(I)$ is a homomorphism

$$
\phi: B=R\left[T_{1}, \ldots, T_{n}\right] \rightarrow \mathscr{R}(I), \quad \phi\left(T_{j}\right)=f_{j} t .
$$

Set $J=\operatorname{kernel}(\phi) ; J$ is a graded ideal of $B, J=\oplus J_{s} . J_{1}$ is the $R$-module of all first order syzygies of $I$, that is, all 1 -forms in the variables $T_{i}, a_{1} T_{1}+\cdots+a_{n} T_{n}$, $\sum a_{i} f_{i}=0$. Similarly $J_{s}$ consists of all first order syzygies of $I^{s}$. Note that $J_{1}$ generates the ideal of relations of the natural map: $B \rightarrow \operatorname{Sym}(I)$. Therefore it follows that $I$ is of linear type if $J=\left(J_{1}\right)$, and that $\delta(I)=J_{2} / B_{1} J_{1}$. Thus we can decide whether $I$ is syzygetic or of linear type as long as we have access to the ideal $J$ and are able to 
make these comparisons. In the last section we shall comment on the use of symbolic computation packages to further these aims.

Finally we shall say that $I$ is generated by a $d$-sequence if it admits a generating set $\left\{x_{1}, \ldots, x_{n}\right\}$ with the property

$\left(x_{1}, \ldots, x_{i}\right): x_{i+1} x_{j}=\left(x_{1}, \ldots, x_{i}\right): x_{j}$, for $i=0, \ldots, n-1$, and $j \geqslant i+1$.

These sequences have seen a variety of uses (see $\left[\mathbf{H} \mathbf{S V}_{\mathbf{2}}\right]$ for a survey); they are characterized by the vanishing of the so-called approximation complexes. In contrast to the case of syzygetic ideals or ideals of linear type, we do not know of a direct method that allows for its detection. This would be desirable-and expected in view of their close relationships that are equivalences in several cases: ideal generated by $d$-sequence $\Rightarrow$ ideal of linear type $\Rightarrow$ syzygetic ideal.

(1.2) LinkAGE. If $I$ and $J$ are ideals of $R$, they are said to be directly linked (notation: $I \sim J$ ) if there exists a regular sequence $\mathbf{x}=\left\{x_{1}, \ldots, x_{g}\right\}$ in $I \cap J$ such that $I=(\mathbf{x}): J$ and $J=(\mathbf{x}): I$. More properly, this notion is sometimes referred to as algebraic linkage. If, in addition, $I$ and $J$ do not have a conmon associated prime, then they are said to be geometrically linked. Although these concepts have their roots in the nineteenth century, impetus for its current usage started out after the appearance of [PS]. Two basic facts were proved in [loc. cit.]: If $I$ is Cohen-Macaulay then $J$ is also Cohen-Macaulay, and if $I$ is perfect, the resolution of $J$ can be obtained in a straightforward manner from that of $I$.

The ideals $I$ and $J$ are said to be linked, if there exists a sequence of ideals $\left\{L_{1}, \ldots, L_{n}\right\}$ such that $I \sim L_{1} \sim \cdots \sim L_{n} \sim J$. The class of all ideals linked to $I$ will be denoted by $\mathbf{L}(I)$. If $n$ above is even, $I$ and $J$ are said to be evenly linked; this subset of $\mathbf{L}(I)$ is denoted by $\mathbf{L}_{e}(I)$. For example, if $I$ is a complete intersection, then $I$ is self-linked, so that $\mathbf{L}_{e}(I)=\mathbf{L}(I)$.

(1.2.1) EXAMPLE. Let $\mathbf{X}$ be a generic, symmetric $3 \times 3$ matrix:

$$
\mathbf{X}=\left[\begin{array}{lll}
x_{1} & x_{2} & x_{3} \\
x_{2} & x_{4} & x_{5} \\
x_{3} & x_{5} & x_{6}
\end{array}\right]
$$

Let $k$ be a field of characteristic 2 and $R$ the ring of polynomials $k\left[x_{1}, \ldots, x_{6}\right]$. The ideal $I$ generated by the $2 \times 2$ minors of $\mathbf{X}$ is self-linked. Also, in any characteristic, $I$ has (SD) but no (SCM) (cf. [Vi]).

The connection of linkage to the conditions above on the depth of the Koszul homology modules is expressed by a theorem of Huneke [ $\left.\mathbf{H u}_{\mathbf{1}}\right]$ : (SD) and (SCM) are invariants of the even linkage class of $I$. (This invariance does not extend to the full linkage class of $I$.) This explains why CM ideals of codimension two and Gorenstein ideals of codimension three are SCM: they are in the linkage class of complete intersections. When the two classes $\mathbf{L}_{e}(I)$ and $\mathbf{L}(I)$ differ, it makes testing whether a given ideal $J$ belongs to $\mathbf{L}(I)$ decisively harder.

There exist other modules attached to $I$ with properties that are invariant for the full linkage class of $I$. Noteworthy are those of [BU], where, for instance, the depth of the twisted conormal module is shown to be an invariant of linkage. They demand, however, ccnsiderably more knowledge of the structure of $I$. By comparison, $H_{1}(I ; R)$ is easy to study, particularly if $I$ is a syzygetic ideal. 
(1.3) Canonical module. If $I$ is a Cohen-Macaulay ideal of height $g$, the canonical module of $S$ is $\operatorname{Ext}^{g}(R / I, R)$; it will be denoted by $W$. Its minimal number of generators, $v(W)$, is the type of $S$. In a minimal free resolution of $I$, $v(W)$ is then the last nonzero Betti number.

(1.3.1) Remark. If $I$ is linked to $J$ through the regular sequence $\mathbf{x}=\left\{x_{1}, \ldots, x_{g}\right\}$, the connection between $W$ and $J$ is straightforward:

$$
W=\operatorname{Ext}^{g}(R / I, R)=\operatorname{Hom}(R / I, R /(\mathbf{x}))=(\mathbf{x}): I /(\mathbf{x})=J /(\mathbf{x}) .
$$

(1.3.2) REMARK. It is useful, whenever possible, to express the fact that one of the Koszul modules is Cohen-Macaulay in terms of the depths of its modules of cycles. Thus, if $v(I)=n$ then depth $\mathbf{Z}_{n-g}=\sup \{d, d-g+2\}$ (cf. [HVV]). In particular, if $n=g+2$, then $I$ is SCM, a fact first observed in [AH]. It also follows that in general $H_{n-g-1}(I ; R)$ is an $S$-module with the $S_{2}$-property of Serre. This will play a role in the duality theory of $H_{*}(\mathbf{K})$.

2. Koszul homology and the canonical module. In this section, $R$ is a Gorenstein local ring of dimension $d \geqslant 4$, and $I$ is a perfect ideal of height 3 . The deviation of an ideal is the difference $v(I)$-height $(I)$. We shall assume that $v(I) \geqslant 4$. Note that ideals of deviation 1 or 2 are SCM.

We examine the role of the divisorial class of $W$-and of its multiples-in relation to the depth of the Koszul homology modules of $I$.

Fixed will be a free resolution of $S$,

$$
0 \rightarrow R^{p} \rightarrow R^{m} \stackrel{\psi}{\rightarrow} R^{n} \rightarrow R \rightarrow S \rightarrow 0 .
$$

The main link to be developed here will be that between certain modules derived from $\mathbf{Z}_{1}=\operatorname{image}(\psi)=$ first-order syzygies of $I$, and the 1-dimensional Koszul homology module $H_{1}=H_{1}(I ; R)$.

The starting point is the identification of the double dual of the module $\Lambda^{2} \mathbf{Z}_{1}$ with $\mathbf{Z}_{2}$, the module of 2-cycles of the associated Koszul complex induced by the algebra structure of $\mathbf{Z}$ (cf. $\left.\left[\mathbf{H S V}_{2}\right]\right)$ :

$$
0 \rightarrow C \rightarrow \Lambda^{2} \mathbf{Z}_{1} \stackrel{\lambda}{\rightarrow} \mathbf{Z}_{2} \rightarrow D \rightarrow 0
$$

where $C$ and $D$ are computed from a presentation of $\Lambda^{2} \mathbf{Z}_{1}$.

Consider the Lebelt-Weyman complex associated to $\Lambda^{2} \mathbf{Z}_{1}[\mathbf{L e}, \mathbf{W e}]$ :

$$
0 \rightarrow D_{2}\left(R^{p}\right) \stackrel{\alpha}{\rightarrow} R^{p} \otimes R^{m} \stackrel{\beta}{\rightarrow} \Lambda^{2} R^{m} \rightarrow \Lambda^{2} \mathbf{Z}_{1} \rightarrow 0 .
$$

This complex is exact by the acyclicity lemma and it follows that $\Lambda^{2} \mathbf{Z}_{1}$ is torsion-free (i.e., $C=0$ ). We examine the homology of its dual,

$$
0 \rightarrow\left(\Lambda^{2} \mathbf{Z}_{1}\right) * \rightarrow\left(\Lambda^{2} R^{m}\right) * \stackrel{\beta^{*}}{\rightarrow}\left(R^{p} \otimes R^{m}\right) * \stackrel{\alpha^{*}}{\rightarrow}\left(D_{2}\left(R^{p}\right)\right)^{*} \rightarrow 0 .
$$

We note that $\left(\Lambda^{2} \mathbf{Z}_{1}\right)^{*}=\mathbf{Z}_{n-3}\left(\mathrm{cf} .\left[\mathbf{H S V}_{2}\right]\right)$, and that $\operatorname{Ext}^{3}\left(\Lambda^{2} \mathbf{Z}_{1}, R\right)=S_{2}(W)$. Moreover, if we set $M=\operatorname{coker}\left(\beta^{*}\right)$, then $C=\operatorname{Ext}^{1}(M, R)$ and $D=\operatorname{Ext}^{2}(M, R)$ (cf. [AB, p. 52]). 
Setting $N=\operatorname{image}\left(\beta^{*}\right)$ and $L=\operatorname{ker}\left(\alpha^{*}\right)$ we obtain the exact sequences

(\#)

$$
0 \rightarrow N \rightarrow L \rightarrow \operatorname{Ext}^{1}\left(\Lambda^{2} \mathbf{Z}_{1}, R\right) \rightarrow 0
$$

and

$$
0 \rightarrow L \rightarrow\left(R^{p} \otimes R^{m}\right)^{*} \rightarrow\left(D_{2}\left(R^{p}\right)\right)^{*} \rightarrow S_{2}(W) \rightarrow 0 .
$$

Because $D$ is a module that vanishes in codimension two or less, we obtain $\operatorname{Ext}^{1}\left(\Lambda^{2} \mathbf{Z}_{1}, R\right)=\operatorname{Ext}^{1}\left(\mathbf{Z}_{2}, R\right)=\operatorname{Ext}^{1}\left(B_{1}, R\right)=\operatorname{Ext}^{3}\left(H_{1}, R\right)$.

In addition we have $D=\operatorname{Ext}^{2}(M, R)=\operatorname{Ext}^{1}(N, R)=\operatorname{Ext}^{1}(L, R)=$ $\operatorname{Ext}^{3}\left(S_{2}(W), R\right)$.

The following embodies several technical aspects of the relationship between $H_{1}(I)$ and $S_{2}(W)$; it expands upon both [HVV, Theorem 2.1] and [VV, Theorem $1.3]$.

(2.1) TheOREM. Let $R$ be a Gorenstein local ring, and let $I$ be a perfect ideal of height 3.

(a) If I is generically a Gorenstein ideal that is syzygetic on the punctured spectrum of $R$ and $W^{*}$ has depth at least 3 , then $I$ is syzygetic.

(b) $H_{1}$ is Cohen-Macaulay if and only if $S_{2}(W)$ is Cohen-Macaulay.

(c) If $I$ is syzygetic (or, more generally, $H_{1}$ is a torsion-free module) then depth $S_{2}(W) \neq d-4$.

(d) If $I$ is generically a Gorenstein ideal and $H_{1}$ is not Cohen-Macaulay then depth $H_{1} \geqslant \operatorname{depth}\left(W^{*}\right)-2$; moreover, if $H_{1}$ is an $S_{2}$-module and $I$ is $S C M$ in codimension one, then depth $S_{2}(W)=$ depth $H_{n-4}-2$.

Proof. (a) First, note that for any $S$-module $M, \operatorname{Ext}^{3}(M, R)=\operatorname{Hom}(M, W)$, so that $\operatorname{Ext}^{3}\left(S_{2}(W), R\right)=\operatorname{Hom}\left(S_{2}(W), W\right)$; given that $W$ can be identified to an ideal of $S$, the last module may be expressed as $\operatorname{Hom}(W \otimes W, W)=W^{*}$. From the sequence (\#) we get that depth $H_{1} \geqslant 1$. It suffices to use the induction hypothesis in the exact sequence of 1.1 .3 to obtain $\delta(I)=0$.

(b) Clearly $H_{1}$ is Cohen-Macaulay if and only if $\mathbf{Z}_{2}$ has depth $d-1$. In this case $D$ is a Cohen-Macaulay module and $\operatorname{Ext}^{3}(D, R)$ is Cohen-Macaulay along with it. But again with depth $\mathbf{Z}_{2}=d-1$, that module is isomorphic to $\operatorname{Ext}^{2}\left(\Lambda^{2} \mathbf{Z}_{1}, R\right)=$ $S_{2}(W)$.

Conversely, if $S_{2}(W)$ is Cohen-Macaulay so is $D$ by the identification above. This implies that depth $\mathbf{Z}_{2} \geqslant d-3$. We show that $\operatorname{Ext}^{i}\left(\mathbf{Z}_{2}, R\right)=0$ for $i=2$ and 3, which will sustain the claim. We have the following exact sequence derived from (\#):

$$
0 \rightarrow \operatorname{Ext}^{2}\left(\mathbf{Z}_{2}, R\right) \rightarrow S_{2}(W) \rightarrow \operatorname{Ext}^{3}\left(\operatorname{Ext}^{3}\left(S_{2}(W), R\right), R\right) \rightarrow \operatorname{Ext}^{3}\left(\mathbf{Z}_{2}, R\right) \rightarrow 0 .
$$

Because $S_{2}(W)$ is Cohen-Macaulay, by duality we have $\operatorname{Ext}^{3}\left(\operatorname{Ext}^{3}\left(S_{2}(W), R\right), R\right)$ $=S_{2}(W)$, so that we must prove that the map between these two copies of $S_{2}(W)$ is an isomorphism. To show $\operatorname{Ext}^{2}\left(\mathbf{Z}_{2}, R\right)=0$ it suffices to localize at primes in the support of $S_{2}(W)$; since such a prime $P$ has height 3 and $\mathbf{Z}_{2}$ is a reflexive module, $\operatorname{Ext}^{2}\left(\mathbf{Z}_{2}, R\right)_{P}=0$. If $\operatorname{Ext}^{3}\left(\mathbf{Z}_{2}, R\right) \neq 0$, it is a module of dimension $d-4$; localizing at a prime $P$ of height 4 the same argument yields a contradiction. 
(c) From the sequences (\#) we have the depth $L=\operatorname{depth} S_{2}(W)+2$. Let us show that depth $L \neq d-2$. Dualizing the first of the sequences, we obtain

$$
0 \rightarrow \operatorname{Ext}^{2}(L, R) \rightarrow \operatorname{Ext}^{2}(N, R) \stackrel{\delta}{\rightarrow} \operatorname{Ext}^{3}\left(\operatorname{Ext}^{3}\left(H_{1}, R\right), R\right) .
$$

But $\operatorname{Ext}^{2}(N, R)=\operatorname{Ext}^{1}\left(\mathbf{Z}_{n-3}, R\right)=\operatorname{Ext}^{1}\left(B_{n-3}, R\right)=H_{1}$, and $\delta$ is the map from $H_{1}$ into its double dual with respect to $W$. Since $H_{1}$ is torsion-free and this map is generically an isomorphism, it follows that $\operatorname{Ext}^{2}(L, R)=0$-which is a contradiction.

(d) We only have to prove the last assertion. Dualize the sequence (\#) to obtain

$$
\begin{aligned}
0 & \rightarrow \operatorname{Ext}^{2}(L, R) \rightarrow \operatorname{Ext}^{2}(N, R)=\operatorname{Ext}^{1}\left(\mathbf{Z}_{n-3}, R\right) \\
& =H_{1} \rightarrow \operatorname{Ext}^{3}\left(\operatorname{Ext}^{3}\left(H_{1}, R\right), R\right) \rightarrow \operatorname{Ext}^{3}(L, R) \rightarrow 0 .
\end{aligned}
$$

The proof will follow from the isomorphism of the mid modules. This is a consequence of the following general fact.

(2.2) Proposition. Let $R$ be a Gorenstein local ring and let I be a Cohen-Macaulay ideal of height $g$, generated by $n$ elements. There exists a canonical homomorphism

$$
H_{n-g-1} \rightarrow \operatorname{Ext}^{g}\left(H_{1}, R\right)
$$

that is an isomorphism whenever I is SCM in codimension one.

Proof. The multiplication in the algebra $H_{*}(\mathbf{K})$ induces a pairing

$$
H_{i} \times H_{n-g-i} \rightarrow H_{n-g}
$$

and therefore a mapping

$$
H_{i} \rightarrow \operatorname{Hom}\left(H_{n-g-i}, H_{n-g}\right) .
$$

As $H_{n-g}$ is the canonical module of $R / I$, the last module may be written as $\operatorname{Ext}^{g}\left(H_{n-g-i}, R\right)$. Herzog proved [ $\left.\mathbf{H e}_{2}\right]$ that this pairing is an isomorphism if $I$ is SCM. The claim here is that for $i=n-g-1$ it suffices that $I$ be SCM in codimension one.

It is clearly enough to prove that $H_{n-g-1}$ is an $S_{2}$-module and that $\operatorname{Ext}^{g}\left(H_{1}, R\right)$ is a torsion-free $S$-module. (Actually it turns out that the last module is always $S_{2}$.) The SCM hypothesis and [loc. cit.] then apply to show the isomorphism.

That $H_{n-g-1}$ is $S_{2}$ was observed in (1.3.2). As for the other module, note that $H_{1}$ has exactly the same associated minimal primes as $R / I$. Indeed, if $P$ is a minimal prime of the support of $H_{1}$, not minimal over $I$, localizing the exact sequence

$$
H_{1} \rightarrow(R / I)^{n} \rightarrow I / I^{2} \rightarrow 0
$$

would say that the image of $H_{1}$ has finite length in $(R / I)_{P}^{n}$, so that it must be 0 . Thus $\left(I / I^{2}\right)_{P}$ is free and $I_{P}$ must be a complete intersection since it has finite projective dimension [V]. But then the localization of $H_{1}$ would be free. The converse is seen in a similar manner.

Let us show that $\operatorname{Ext}^{g}\left(H_{1}, R\right)$ is a torsion-free $R / I$-module. Let $a$ be an element regular on $R / I$. Denote by $L$ the submodule of $H_{1}$ annihilated by some power of $a$, and set $M=H_{1} / L$. $L$ has codimension at least $g+1$, so that applying the functor 
$\operatorname{Hom}(-, R)$ to $M$ we obtain the exact sequence

$$
0 \rightarrow \operatorname{Ext}^{g}(M, R) \rightarrow \operatorname{Ext}^{g}\left(H_{1}, R\right) \rightarrow \operatorname{Ext}^{g}(L, R)=0 .
$$

It suffices to show that $\operatorname{Ext}^{g}(M, R)$ is torsion-free. Since $a$ is regular on $M$, from

$$
0 \rightarrow M \stackrel{a}{\rightarrow} M \rightarrow M / a M \rightarrow 0
$$

we obtain that $a$ is regular on $\operatorname{Ext}^{g}(M, R)$, as the support of $M / a M$ has codimension $g+1$ and $\operatorname{Ext}^{g}(M / a M, R)=0$.

The argument also shows that $\operatorname{Ext}^{g}(M, R) / a \operatorname{Ext}^{g}(M, R)$ embeds into $\operatorname{Ext}^{g+1}(M / a M, R)$. It is easy to prove that the associated minimal primes of $M / a M$ are the same as those of $R /(I, a)$, so that the argument above on $H_{1}$, as applied to $M / a M$, would show $\operatorname{Ext}^{g+1}(M / a M, R)$ is a torsion-free $R /(I, a)$-module. Therefore $\operatorname{Ext}^{g}\left(H_{1}, R\right)$ is an $S_{2}$-module.

(2.3) EXAMPLE. Let $I$ be the ideal generated by the $(k-1)$-sized minors of a generic, symmetric $k \times k$ matrix, $k \geqslant 3$. ( $R$ is the polynomial ring generated by the entries of the matrix over a field of characteristic $\neq 2$.) $I$ is a Cohen-Macaulay ideal of height 3. Furthermore, $W^{*}=W$ (cf. [Go]). It follows therefore that $I$ is syzygetic and that depth $\left(H_{1}\right) \geqslant d-5$. The equality follows from the following argument. As in the proof of (b) we must show that $\operatorname{Ext}^{3}\left(\mathbf{Z}_{2}, R\right) \neq 0$. But here $\operatorname{Ext}^{3}(D, R)=$ $\operatorname{Ext}^{3}(W, R)=S$ and we cannot have a surjection $S_{2}(W) \rightarrow S$ unless $W$ itself admits a free summand, which is only the case for Gorenstein ideals. If $k=3, I$ is known to be generated by a $d$-sequence [HVV].

We have come to the main result of the section.

(2.4) TheOREM. Let I be a perfect ideal of codimension three and let $J$ be an ideal linked to I. If $H_{1}(I)$ is Cohen-Macaulay, then $H_{1}(J)$ is also Cohen-Macaulay.

We first translate the condition on $H_{1}(I)$ into yet another one on the depth of the symmetric square of $I$ itself.

(2.5) LemMA. If I is perfect of codimension three then $H_{1}(I)$ is Cohen-Macaulay if and only if depth $S_{2}(I) \geqslant d-3$.

Proof. We use another of the complexes of [We], that corresponding to $S_{2}(I)$, which for convenience we split into two pieces:

$$
\begin{aligned}
& 0 \rightarrow D_{2}\left(R^{p}\right) \rightarrow R^{p} \otimes R^{m} \rightarrow R^{p} \otimes R^{n} \oplus \Lambda^{2}\left(R^{m}\right) \rightarrow L \rightarrow 0, \\
& 0 \rightarrow K \rightarrow R^{p} \otimes R^{m} \rightarrow S_{2}\left(R^{n}\right) \rightarrow S_{2}(I) \rightarrow 0,
\end{aligned}
$$

where $L \subset K$; denote $K / L=M$.

Because the entire free complex is minimal, it is not exact: it suffices to test at a minimal prime of $I$. On the other hand, since it is split exact in codimension less than three, it follows that both pieces of the complex are exact by the acyclicity lemma.

Note that $\operatorname{Ext}^{2}(L, R)=S_{2}(W)$ and $\operatorname{pd}_{R}(L)=2$. We look at the long cohomology sequence associated to $M=K / L$. Since $M$ vanishes in codimension less than three, we have

$$
0 \rightarrow \operatorname{Ext}^{2}(K, R) \rightarrow \operatorname{Ext}^{2}(L, R) \rightarrow \operatorname{Ext}^{3}(M, R) \rightarrow \operatorname{Ext}^{3}(K, R) \rightarrow 0 .
$$


Assume that $H_{1}(I)$ is Cohen-Macaulay; by Theorem 2.1, $S_{2}(W)$ is CohenMacaulay. Since $\operatorname{Ext}^{2}(K, R)=\operatorname{Ext}^{4}\left(S_{2}(I), R\right)$, its support has codimension at least four, so that it must be trivial as it lies in $S_{2}(W)$. To show that $\operatorname{Ext}^{3}(L, R)=$ $\operatorname{Ext}^{5}\left(S_{2}(I), R\right)$ also vanishes, note that its support has codimension at least five. We may further assume $d \geqslant 5$ and that it vanishes on the punctured spectrum.

Since $S_{2}(W) \subset \operatorname{Ext}^{3}(M, R)$, the codimension of the support of $M$ is exactly three. It follows that $\operatorname{Ext}^{3}(M, R)$ has depth at least one. Indeed, if $M^{\prime}=H_{m}^{0}(M)$, we easily get that $\operatorname{Ext}^{3}(M, R)=\operatorname{Ext}^{3}\left(M / M^{\prime}, R\right)$. Because $\mathbf{m}$ is not associated to $M / M^{\prime}$, there exists an element $a \in \mathbf{m}$ regular on $M / M^{\prime}$. Using then the Extsequence associated to the exact sequence

$$
0 \rightarrow M / M^{\prime} \underset{\phi}{\rightarrow} M / M^{\prime} \rightarrow G \rightarrow 0,
$$

where $\phi$ is a multiplication by $a$, it follows that $a$ is also regular on $\operatorname{Ext}^{3}(M, R)$. Taking this into the exact sequence of $\operatorname{Ext}^{5}\left(S_{2}(I), R\right)$, we get that this module must have depth at least one-which is a contradiction unless it is trivial.

We therefore obtain that $S_{2}(W)=\operatorname{Ext}^{3}(M, R)$. Since $M$ has depth at least 2 , it follows from the duality theorem of [HO] that $M$ itself is Cohen-Macaulay. This shows that depth $K \geqslant d-3$ and that $\operatorname{Ext}^{i}(K, R)=0$ for $i=2$ and 3 . Thus depth $K \geqslant d-1$, and depth $S_{2}(I) \geqslant d-3$.

The converse is clear from these arguments.

Proof of (2.4). If $J$ is linked to $I$ through the regular sequence $\mathbf{x}=\left\{x_{1}, x_{2}, x_{3}\right\}$, the canonical module of $R / J$ is simply $W=I / L, L=(\mathbf{x})$. We then have the induced sequence of symmetric squares:

$$
0 \rightarrow I \cdot L \rightarrow S_{2}(I) \rightarrow S_{2}(W) \rightarrow 0 .
$$

Since $H_{1}(I)$ is Cohen-Macaulay, $S_{2}(I)$ has depth $\geqslant d-3$. The module $I \cdot L$ maps isomorphically onto the product $I L$. Indeed, if $z=x_{1} \cdot f_{1}+x_{2} \cdot f_{2}+x_{3} \cdot f_{3}$ is an element of the kernel, $f_{1} x_{1}+f_{2} x_{2}+f_{3} x_{3}=0$ implies that $f_{1}=a x_{2}+b x_{3}, f_{2}=$ $-a x_{1}+c x_{3}, f_{3}=-a x_{1}-c x_{2}$, so that $z=0$. On the other hand, as $L / L^{2}$ is a free $R / L$-module, $L / L^{2} \otimes R / I=L / I L$ is a free $R / I$-module and therefore depth $I L=d-2$. It follows that depth $S_{2}(W) \geqslant d-3$, and it is thus a CohenMacaulay module. Now use (2.1).

For these ideals, the condition " $H_{1}$ is Cohen-Macaulay" is retained along the full linkage class of $I$ rather than just the even linkage subclass predicated by the general theory. As pointed out in [ $\mathbf{H u}_{2}$, Example 2.5], this condition fails already for ideals of codimension four.

A testing ground for the condition " $H_{1}$ is Cohen-Macaulay" is provided by the ideals with pure resolutions. These are homogeneous ideals of $k\left[x_{1}, \ldots, x_{n}\right]$ whose (graded) projective resolutions have the form $(g=3)$

$$
0 \rightarrow R^{b_{3}}(-d-a-b) \rightarrow R^{b_{2}}(-d-a) \rightarrow R^{b_{1}}(-d) \rightarrow R \rightarrow S \rightarrow 0 .
$$

For a graded module $M$, the twisted module $M(r)$ is defined by $M(r)_{n}=M_{r+n}$. For Cohen-Macaulay modules with pure resolutions the Betti numbers $b_{i}$ are given in terms of the twists of the resolution (and $b_{0}$ ) [HK]. Villarreal considered the following question [Vi]. 
Conjecture (A). Let $I$ be a homogeneous ideal of $R$ of codimension 3 , and deviation at least 3. Assume that $I$ is not a Gorenstein ideal and is generically a complete intersection. If the resolution of $S$ is pure then $I$ is not SCM.

Using a semi-numerical analysis of various Hilbert functions, carried out in a computer, a large part of the conjecture was proved-likely more than half of all possible cases:

(2.6) THEOREM [Vi]. Let I be a codimension 3 Cohen-Macaulay ideal as above with a pure resolution. If $a \geqslant b$ and $b_{1} \geqslant 6$, then $H_{1}(I)$, the first Koszul homology module, is not Cohen-Macaulay.

This takes care of all ideals with fewer than 24 generators, with the exception of $b_{1}=8$. There are examples of ideals with 8 generators and $H_{1}$ Cohen-Macaulay, as constructed in [KMU, Example 2.6]. This ideal has type 2, so would have $H_{1}$ Cohen-Macaulay by Corollary 2.9. It was also shown in [Vi] that $H_{2}$ is not Cohen-Macaulay (see also 4.2.4).

Theorem 2.4 can be used to further strengthen the conjecture. The act of linking an ideal with a pure resolution through a homogeneous regular sequence returns an ideal (not necessarily with a pure resolution) whose numerical data is known, so that a similar analysis can be undertaken. This has extended somewhat the range of the conjecture.

If the type of the ideal $I$ is small ( $p=2,3)$, some additional information about $H_{1}$ is available. To get at this consider the following construction. Let $M$ be a torsion-free module over a ring $S$, generated by $p$ elements,

$$
0 \rightarrow L \rightarrow S^{p} \rightarrow M \rightarrow 0 .
$$

It induces the commutative diagram

$$
\begin{gathered}
0 \rightarrow \Lambda^{2} L \rightarrow \quad L \otimes S^{p} \stackrel{\alpha}{\rightarrow} S_{2}\left(S^{p}\right) \rightarrow S_{2}(M) \rightarrow 0 \\
\beta \searrow \nearrow \gamma \\
S^{p} \otimes S^{p}
\end{gathered}
$$

with canonical maps.

Applying the snake lemma to the composite $\alpha=\gamma \cdot \beta$, we obtain the exact sequence

$$
0 \rightarrow \operatorname{ker}(\beta) \rightarrow \operatorname{ker}(\alpha) \rightarrow \operatorname{ker}(\gamma) \rightarrow \operatorname{coker}(\beta) \rightarrow \operatorname{coker}(\alpha) \rightarrow \operatorname{coker}(\gamma) \rightarrow 0 .
$$

One has that $\operatorname{ker}(\beta)=\operatorname{coker}(\gamma)=0, \operatorname{coker}(\beta)=M^{p}$ and $\operatorname{coker}(\gamma)=S^{r}, r=\left[\begin{array}{l}p \\ 2\end{array}\right]$. The map from $S^{r}$ into $M^{p}$ can be viewed as the 2-dimensional Koszul map defined by the generating set of $M$ used in the presentation.

We may write the resulting complex as

$$
0 \rightarrow\left(\Lambda^{2} L\right)^{* *} \rightarrow S^{r} \rightarrow M^{p} \rightarrow S_{2}(M) \rightarrow 0 .
$$

If $p=2,\left(\Lambda^{2} L\right)^{* *}=0$, and one can use the similar construction for an arbitrary symmetric power of $M$ and obtain the exact sequence $(t \geqslant 2)$

$$
0 \rightarrow S^{t-1} \rightarrow M^{t} \rightarrow S_{t}(M) \rightarrow 0 .
$$


We first point out an implication of this sequence to explain a peculiarity of two-generated divisorial ideals in normal domains.

(2.7) THEOREM. Let $R$ be a Noetherian, normal local domain and let I be a divisorial ideal generated by two elements. Assume that for each prime ideal $P$ such that depth $R_{P}=2$ the ideal $I_{P}$ is principal. Then the divisor class of I cannot have finite order.

Proof. The hypotheses, when taken into the sequence, imply that $S_{t}(I)$ is a divisorial ideal. It cannot be principal unless $I$ itself is such.

(2.8) Proposition. Let $R$ be a local Gorenstein ring and let I be a perfect ideal of type 2. Then

(a) $S_{2}(W)$ is Cohen-Macaulay.

(b) depth $S_{t}(W)=\operatorname{depth} S-1$, for $t>2$.

Proof. (a) Let $x$ and $y$ be the generators of $W$. The exact sequence to consider is

$$
0 \rightarrow S=S e \rightarrow W \oplus W \rightarrow S_{2}(W) \rightarrow 0,
$$

where $e$ maps to $(-y, x)$.

If we tensor the sequence by $S^{\prime}=S /(\mathbf{x})$, where $\mathbf{x}$ is a system of parameters of $S$, we still get an embedding of $S^{\prime}$ into $W^{\prime} \oplus W^{\prime}$, as $W^{\prime}$ is the canonical module of $S^{\prime}$ and is therefore a faithful module. Therefore $\operatorname{Tor}_{1}\left(S^{\prime}, S_{2}(W)\right)=0$, so that $S_{2}(W)$ is a Cohen-Macaulay module.

(b) If $S_{t}(W)$ is Cohen-Macaulay, applying $\operatorname{Hom}(-, W)$ to the sequence

$$
0 \rightarrow S^{t-1} \rightarrow W^{t} \rightarrow S_{t}(W) \rightarrow 0
$$

we obtain the surjection

$$
\operatorname{Hom}\left(W^{t}, W\right)=S^{t} \rightarrow \operatorname{Hom}\left(S^{t-1}, W\right)=W^{t-1},
$$

which is a contradiction since $v\left(W^{t-1}\right)=2(t-1)$.

(2.9) Corollary. Let I be a perfect ideal of height 3 and type 2. Then $H_{1}(I)$ is Cohen-Macaulay.

If $p=3$, the sequence obtained is

$$
0 \rightarrow K \rightarrow S^{3} \rightarrow W^{3} \rightarrow S_{2}(W) \rightarrow 0 .
$$

Dualizing the exact sequence and taking into account the skew symmetry of the map $\phi$, we also obtain that $K=D=\operatorname{Ext}^{3}\left(S_{2}(W), R\right)$. Example 2.3 for $k=3$ has $p=3$ so that $S_{2}(W)$ is not always Cohen-Macaulay. Nevertheless we have:

(2.10) Proposition. Let I be a perfect ideal of height 3 and type 3. Suppose that $S_{2}(W)$ is a reflexive $S$-module and that in each localization (still denoted by $R$ ) depth $H_{1}(I) \geqslant(1 / 2)(\operatorname{dim} R-1)$. Then $S_{2}(W)$ is Cohen-Macaulay.

Proof. By hypothesis depth $\mathbf{Z}_{2} \geqslant(1 / 2)(\operatorname{dim} R-1)+2$, so that depth $D \geqslant$ $(1 / 2)(\operatorname{dim} R-1)+2$ as well. The sequence above implies that depth $S_{2}(W) \geqslant$ depth $K-2$. We therefore have the inequality

$$
\operatorname{depth} S_{2}(W)+\operatorname{depth} \operatorname{Hom}\left(S_{2}(W), W\right) \geqslant \operatorname{dim} S+2,
$$


which by the duality theorem of [HO] (see also [Hu $\mathbf{H}_{2}$, Lemma 5.5]) means that $S_{2}(W)$ is Cohen-Macaulay.

3. Gorenstein ideals of codimension four. Let $R$ be a Gorenstein local ring and let $I$ be a Gorenstein ideal of codimension four. We develop more fully the connection between the Cohen-Macaulayness of the modules $I / I^{2}$ and the Koszul homology of $I$ treated in [VV]. The relationship described there between $I / I^{2}$ and $H_{1}(I)$ is shown to hold both ways. It turns out that the closer connection is that between $I / I^{2}$ and both $H_{1}(I)$ and the penultimate Koszul homology module. It permits the determination - for syzygetic ideals - of the codimension of the Cohen-Macaulay locus of $I / I^{2}$. One corollary says that syzygetic ideals generated by seven elements have the property that $I / I^{2}$ is Cohen-Macaulay as long as $H_{1}(I)$ is an $S_{2}$-module, that is provided $I / I^{2}$ is a torsion-free $R / I$-module. Some examples are examined in detail.

(3.1) THEOREM. Let $R$ be a Gorenstein local ring, and let $I$ be a Gorenstein ideal of codimension four. Then $H_{1}(I)$ is Cohen-Macaulay if and only if $I / I^{2}$ is CohenMacaulay.

Proof. Fix a minimal free resolution of $S=R / I$ :

$$
0 \rightarrow R \stackrel{\alpha}{\rightarrow} R^{n} \stackrel{\beta}{\rightarrow} R^{m} \rightarrow R^{n} \stackrel{\theta}{\rightarrow} R \rightarrow S \rightarrow 0
$$

Set $\mathbf{a}=\alpha(1)=x_{1} e_{1}+\cdots+x_{n} e_{n}$, with the $e_{i}$ 's forming a basis of $R^{n}$ and $\mathbf{x}=\left\{x_{1}, \ldots, x_{n}\right\}$ a minimal generating set of $I$.

To connect the two modules we use the module of first-order syzygies of $I$, $\mathbf{Z}_{1}=$ image $(\theta)$. There exists, as pointed out in $\$ 2$, a canonical identification of the double dual of $\Lambda^{2} \mathbf{Z}_{1}$ with $\mathbf{Z}_{2}$ (2-cycles of the Koszul complex built on the chosen generators of $I$ ):

$$
\text { (\#) } \quad 0 \rightarrow C=\operatorname{ker}(\lambda) \rightarrow \Lambda^{2} \mathbf{Z}_{1} \stackrel{\lambda}{\rightarrow} \mathbf{Z}_{2} \rightarrow D=\operatorname{coker}(\lambda) \rightarrow 0 .
$$

$H_{1}(I)$ Cohen-Macaulay is obviously equivalent to depth $\mathbf{Z}_{2}=d-2$. We now link $I / I^{2}$ to $D$ and the structure of the sequence. For this we use the free resolution of $\Lambda^{2} \mathbf{Z}_{1}$; it is obtainable using the complexes of [Le, We]:

$$
\text { (\#) } 0 \rightarrow R \otimes R^{n} \stackrel{\phi}{\rightarrow} R \otimes R^{m} \oplus D_{2}\left(R^{n}\right) \stackrel{\psi}{\rightarrow} R^{m} \otimes R^{n} \stackrel{\gamma}{\rightarrow} \Lambda^{2} R^{m} \rightarrow \Lambda^{2} \mathbf{Z}_{1} \rightarrow 0 .
$$

Here the nature of the map $\phi=\left(\phi_{1}, \phi_{2}\right)$ is important:

$$
\phi_{1}=1 \otimes \beta \text { and } \phi_{2}\left(1 \otimes e_{i}\right)=-\mathbf{a} e_{i} \text {. }
$$

Since $\operatorname{pd}_{R}\left(\Lambda^{2} \mathbf{Z}_{1}\right)=3$, its associated primes have height at most 3; as $\mathbf{Z}_{1}$ is free in codimension less than 4 , it follows that $\Lambda^{2} \mathbf{Z}_{1}$ is torsion-free and therefore $C=0$. On the other hand, as in [VV] it follows the $\operatorname{Ext}^{3}\left(\Lambda^{2} \mathbf{Z}_{1}, R\right)=I / I^{2}$. Considering the cohomology sequence associated to (\#) and taking into account that $D$ is a module of codimenion at least four, we get

$$
0 \rightarrow \operatorname{Ext}^{3}\left(\mathbf{Z}_{2}, R\right) \rightarrow I / I^{2} \rightarrow \operatorname{Ext}^{4}(D, R) \rightarrow \operatorname{Ext}^{4}\left(\mathbf{Z}_{2}, R\right) \rightarrow 0 .
$$


We may further clarify this exact sequence when it is observed that $\operatorname{Ext}^{4}\left(\mathbf{Z}_{2}, R\right)=$ $\operatorname{Ext}^{5}\left(B_{1}, R\right)=\operatorname{Ext}^{6}\left(H_{1}, R\right)$, and similarly $\operatorname{Ext}^{3}\left(\mathbf{Z}_{2}, R\right)=\operatorname{Ext}^{5}\left(H_{1}, R\right)$.

To prove the assertions, suppose first $H_{1}$ is Cohen-Macaulay: then $D$ is a module of depth $d-4$ and codimension at least 4; it must then be Cohen-Macaulay and $\operatorname{Ext}^{4}(D, R)$ along with it. Because the end modules vanish in the last exact sequence, $I / I^{2}$ is Cohen-Macaulay.

Conversely, if $I / I^{2}$ is Cohen-Macaulay we may localize first at one of its associated primes to conclude that $\operatorname{Ext}^{3}\left(\mathbf{Z}_{2}, R\right)$ vanishes. It suffices now to show that $D$ and its dual, $\operatorname{Ext}^{4}(D, R)$, are $S_{2}$-modules and that $D=\operatorname{Ext}^{4}\left(\operatorname{Ext}^{4}(D, R), R\right)$.

That $D$ is an $S_{2}$-module follows from (\#): It suffices to localize at any prime in the support of $D$ to ascertain this. But there exists a canonical map from $D$ into its double dual (cf. [KH]); the isomorphism follows from the $S_{2}$-condition (cf. [loc. cit.]).

(3.2) REMARKS. (a) One of the motivations for (3.1) was the need for an unrestricted version of the main theorem of $[\mathbf{H M}]$. They proved that if $I$ is a Gorenstein ideal of codimension 4 , generated by six elements, that is generically a complete intersection then it is a hypersurface section of a Gorenstein ideal of codimension three provided $I / I^{2}$ is Cohen-Macaulay. (The proof excepts characteristic 2.) As noticed earlier Cohen-Macaulay ideals of deviation 1 or 2 are always strongly Cohen-Macaulay.

(b) Herzog (cf. $\left[\mathbf{H e}_{\mathbf{1}}\right]$ ) showed that if $I$ is a Gorenstein ideal of codimension 3, then $I / I^{2}$ is Cohen-Macaulay. Of course the explanation for this is that they are in the linkage class of a complete intersection $\left(\mathbf{c f}\right.$. $\left.\left[\mathbf{H u}_{1}\right]\right)$. Here is yet another way to derive this. By Theorem $2.1 H_{1}$ is a Cohen-Macaulay module. Add to $I$ an indeterminate $x, J=(I, x)$; this ideal (over the ring $R[x]$ ) has the same Koszul homology as $I$ (over $R$ ). Since $J$ is a Gorenstein ideal and $J / J^{2}=I / I^{2} \oplus R / I$, the assertion is proved.

(3.3) ExAmples. (a) Let $k$ be a field and let $X$ be the generic matrix $\left[x_{i j}, 1 \leqslant i \leqslant\right.$ $3,1 \leqslant j \leqslant 4]$. Let $I$ be the ideal generated by the $3 \times 3$ minors of $X$ and the following three polynomials ( $T_{j}$ 's are new indeterminates): $\sum_{j=1}^{4} x_{i j} T_{j}, i=1,2,3$. If $G$ is the associated graded ring of the ideal of $3 \times 3$ minors of $X, I$ is the kernel of the natural surjection $k\left[x_{i j}\right.$ 's, $T_{j}$ 's] $\rightarrow G$. According to $\left[\mathbf{H S V}_{\mathbf{1}}\right] I$ is a Gorenstein prime ideal of height 4 .

To prove that $I / I^{2}$ is Cohen-Macaulay, we specialize the matrix $X$ down to

$$
\left[\begin{array}{llll}
x & 0 & 0 & w \\
0 & y & 0 & w \\
x & 0 & z & 0
\end{array}\right]
$$

and make $T_{1}=x, T_{2}=y, T_{3}=-z, T_{4}=-w$.

One then verifies (see 5.11) the equality of dimensions

$$
l\left(I / I^{2}\right)=\operatorname{rank}\left(I / I^{2}\right) \cdot l(R / I)=4 \times 17=68 .
$$

The criterion of $\left[\mathrm{He}_{\mathbf{1}}\right]$ then says that $I / I^{2}$ is Cohen-Macaulay. It is also the case that the ideal $I$ is generated by a $d$-sequence. 
(b) The other type of Gorenstein ideal of height 4 that arises as a presentation ideal of the associated graded ring is obtained as follows. Let $J$ be a Cohen-Macaulay ideal of codimenion three that is an almost complete intersection and is generically a complete intersection. Let $I$ be the presentation ideal

$$
0 \rightarrow I \rightarrow B=R\left[T_{1}, \ldots, T_{4}\right] \rightarrow \mathrm{gr}_{J}(R) \rightarrow 0 .
$$

$I$ is then a Gorenstein ideal [HVS $\mathbf{H}_{1}$.

(c) Other Gorenstein ideals of height 4 are the defining ideals of monomial rings of a symmetric semigroup generated by five elements, $R=k\left[t^{a}, t^{b}, t^{c}, t^{d}, t^{e}\right]$. For $(6,7,8,9,10)$ it is easy to verify that $I / I^{2}$ is not Cohen-Macaulay.

To see more closely the connection between $I / I^{2}$ and the Koszul homology of $I$ we must examine the homology of the dual of $(\#)$ :

$$
\begin{aligned}
(\# \#) \quad 0 & \rightarrow\left(\Lambda^{2} \mathbf{Z}_{1}\right)^{*} \rightarrow\left(\Lambda^{2} R^{m}\right) * \stackrel{\gamma^{*}}{\rightarrow}\left(R^{m} \otimes R^{n}\right)^{*} \\
& \stackrel{\psi^{*}}{\rightarrow}\left(R \otimes R^{m} \oplus D^{2}\left(R^{n}\right)\right)^{*} \stackrel{\phi^{*}}{\rightarrow}\left(R \otimes R^{n}\right)^{*} \rightarrow I / I^{2} \rightarrow 0 .
\end{aligned}
$$

We single out the cohomology in dimensions 1 and 2 .

$$
0 \rightarrow \operatorname{image}\left(\gamma^{*}\right) \rightarrow \operatorname{ker}\left(\psi^{*}\right) \rightarrow \operatorname{Ext}^{1}\left(\Lambda^{2} \mathbf{Z}_{1}, R\right) \rightarrow 0,
$$

and

$$
0 \rightarrow \operatorname{image}\left(\psi^{*}\right) \rightarrow \operatorname{ker}\left(\phi^{*}\right) \rightarrow \operatorname{Ext}^{2}\left(\Lambda^{2} \mathbf{Z}_{1}, R\right) \rightarrow 0 .
$$

In view of the identification $\left(\Lambda^{2} \mathbf{Z}_{1}\right)^{*}=\mathbf{Z}_{n-3}$, image $\left(\gamma^{*}\right)$ has depth $d-3$. On the other hand, $\operatorname{Ext}^{1}\left(\Lambda^{2} \mathbf{Z}_{1}, R\right)=\operatorname{Ext}^{1}\left(\mathbf{Z}_{2}, R\right)=\operatorname{Ext}^{2}\left(B_{1}, R\right)=\operatorname{Ext}^{2}\left(\mathbf{Z}_{1}, R\right)=$ $\operatorname{Ext}^{3}(I, R)=R / I$, where we have repeatedly used that the modules $\operatorname{Ext}^{i}\left(\Lambda^{2} \mathbf{Z}_{1}, R\right)$, $i=1$ or 2 , have codimension at least 4 .

Thus far we have that depth $\operatorname{ker}\left(\psi^{*}\right) \geqslant d-4$. However by checking the cohomology sequence associated to $\operatorname{Hom}(-, R)$, when applied to $(*)$ and making use of the identification $\left.\operatorname{Ext}^{3}\left(\operatorname{image}(\gamma)^{*}\right), R\right)=\operatorname{Ext}^{2}\left(\mathbf{Z}_{n-3}, R\right)=R / I=\operatorname{Ext}^{4}(R / I, R)$, we conclude that depth $\operatorname{ker}\left(\psi^{*}\right)=d-2$.

In an entirely similar manner, $\operatorname{Ext}^{2}\left(\Lambda^{2} \mathbf{Z}_{1}, R\right)=\operatorname{Ext}^{4}\left(H_{1}, R\right)$.

(3.4) THEOREM. Let I be a Gorenstein ideal of height four that is SCM in codimension at most one. If $I$ is syzygetic, $I / I^{2}$ is torsion-free, and in each localization ( still denoted by $R$ ) depth $H_{1} \geqslant(\operatorname{dim} R-3) / 2$, then $I / I^{2}$ is Cohen-Macaulay.

Proof. Since $I$ is syzygetic and $I / I^{2}$ is torsion-free, $H_{1}$ is an $S_{2}$-module. We may assume that $H_{1}$ is Cohen-Macaulay on the punctured spectrum of $S$. On account of (3.1) and the remark above, we may assume that $d=\operatorname{dim} R \geqslant 7$. Appealing to [HO], we must show that

$$
\text { depth } H_{1}+\operatorname{depth} H_{n-5} \geqslant \operatorname{dim} S+2=d-2 \text {. }
$$

If depth $H_{1} \geqslant(d-3) / 2$, depth $I / I^{2} \geqslant(d-5) / 2$, and therefore $\operatorname{depth} \operatorname{ker}\left(\phi^{*}\right) \geqslant$ $(d-5) / 2+2$. All that remains to do is the identification of $\operatorname{Ext}^{4}\left(H_{1}, R\right)$ with $H_{n-5}$; this however follows from Proposition 2.2. 
We shall cast (3.4) in terms of the codimension of the non-Cohen-Macaulay locus of $I / I^{2}$.

(3.5) Corollary. Let I be a Gorenstein ideal of codimension four. If I is syzygetic and $I / I^{2}$ is torsion-free, then the codimension of the non-Cohen-Macaulay locus of $I / I^{2}$ is less than $(d-5) / 2$.

If $n=7$ Theorem 3.4 is even sharper.

(3.6) TheOREM. Let I be a Gorenstein ideal of height 4 and generated by seven elements. If $I$ is syzygetic and $I / I^{2}$ has no associated prime ideal of codimension 6 then $I / I^{2}$ is Cohen-Macaulay.

Proof. We may assume $d \geqslant 7$. First note that depth $\mathbf{Z}_{2}=1+\operatorname{depth} B_{1}=2+$ depth $H_{1} \geqslant 3$. Since depth $\mathbf{Z}_{3}=d-2$, depth $H_{2} \geqslant 3$, and therefore depth $\operatorname{ker}\left(\phi^{*}\right) \geqslant$ 3 ; this shows that depth $I / I^{2} \geqslant 1$ and thus depth $H_{1} \geqslant 2$. If $d=7$ it implies that $\mathrm{H}_{2}$ is Cohen-Macaulay, so that its dual $H_{1}$ is also Cohen-Macaulay. Assume then $d>7$. In this case depth $H_{2} \geqslant 4$. We claim that this implies that depth $I / I^{2} \geqslant 2$. It suffices to take this estimate into the exact sequence $(* *)$. Each iteration of the argument raises the depth of $I / I^{2}$ until it is shown to be Cohen-Macaulay.

The next result was prompted by a question of Huneke. In $\left[\mathbf{H u}_{3}\right]$ he showed that if $I$ is the ideal generated by the submaximal minors of a generic $n \times n$ matrix $(n \geqslant 3)$ then $I$ is of linear type. His question was whether $I$ is actually generated by a $d$-sequence. The set-up above helps to identify one obstruction for this to happen. For these ideals the answer to the question is then negatively settled. This may be the first example of a Cohen-Macaulay prime ideal of a regular ring that is of linear type but cannot be generated by a $d$-sequence.

(3.7) THEOREM. Let I be a Gorenstein ideal of codimension four. If I is generated by a d-sequence then $I / I^{2}$ is a reflexive $R / I$-module.

Proof. If $d=5$ or 6 , then $I$ has deviation at most two, so it will be strongly Cohen-Macaulay. Assume $d \geqslant 7$. We make use of the acyclicity of the $M$-complex:

$$
\begin{aligned}
0 & \rightarrow H_{n-4} \otimes C[n-4] \\
& \rightarrow \cdots \rightarrow H_{2} \otimes C[-2] \rightarrow H_{1} \otimes C[-1] \rightarrow C \rightarrow \operatorname{Sym}\left(I / I^{2}\right) \rightarrow 0 .
\end{aligned}
$$

It follows from this exact sequence that depth $H_{i}>0$ for each dimension, and in particular that depth $B_{n-6} \geqslant 2$ and thus depth $\mathbf{Z}_{n-5} \geqslant 3$. Since depth $B_{n-5}=d-3$ $\geqslant 4$, depth $H_{n-5} \geqslant 3$, so that from $(* *)$ we get depth $I / I^{2} \geqslant 1$ and therefore depth $H_{1} \geqslant 2$. Going back into the approximation complex with this estimate we get inductively that all the Koszul homology modules have depth at least 2. Another round of the argument (only needed if $d>7$ ) says that depth $\mathbf{Z}_{n-5} \geqslant 4$, and-as depth $B_{n-5} \geqslant 5$-depth $I / I^{2} \geqslant 2$.

This result suggests that it is not unlikely that if $I$ is generated by a $d$-sequence then $I / I^{2}$ is in fact Cohen-Macaulay. 
4. The conormal module. This section contains several complements to the previous ones, making use of the explicitness of certain Koszul modules to settle a number of questions on the Cohen-Macaulayness of the conormal module and the vanishing of some deformation modules.

We make some observations on what has been a puzzling behavior of the Cohen-Macaulayness of the square of a Cohen-Macaulay ideal of height 3. We denote by

$$
0 \rightarrow F_{3} \rightarrow F_{2} \rightarrow F_{1} \rightarrow R \rightarrow S=R / I \rightarrow 0
$$

a minimal graded resolution of $S$, with Betti numbers $b_{3}, b_{2}$, and $b_{1}$.

To give a proper frame we formulate the following:

(4.1) Conjecture (B). Let $I$ be a Cohen-Macaulay ideal. If $I$ is syzygetic and $I / I^{2}$ is Cohen-Macaulay then $I$ is a Gorenstein ideal.

For such an ideal we are postulating that

$$
0 \rightarrow H_{1} \rightarrow S^{n} \rightarrow I / I^{2} \rightarrow 0
$$

is an exact sequence of Cohen-Macaulay modules. This situation could also be paraphrased by saying that the projective dimension of the symmetric square $S_{2}(I)$ is $g-1$.

One of the known cases of this question can be traced to the following argument of Kunz [K]. If $I$ is an almost complete intersection-that is, has deviation at most one-then it must be a complete intersection. Indeed, if the deviation is one, $H_{1}$ is the canonical module $W$ of $S$; applying $\operatorname{Hom}(-, W)$ to the sequence gives that $W$ is free.

We shall key on the codimension three case, in fact on the ideals that pass through the cracks of the Conjecture (A) of $\S 2$, although extensions to more general graded modules are possible. Note that the condition that $\operatorname{pd}_{R}\left(S_{2}(I)\right)=2$ is reflected by the exact sequences of Lemma 2.5 as follows: $K$ is a free module, and $M=W^{\wedge}=$ $\operatorname{Ext}^{3}\left(S_{2}(W), R\right)$.

Denoting by $\mathscr{F}(\cdot)$ the Hilbert series of a graded module $(\cdot)$, we have the following expression for the Hilbert function of $K$ :

$$
\mathscr{F}(K)=\mathscr{F}\left(F_{3} \otimes F_{1}\right)+\mathscr{F}\left(\Lambda^{2} F_{2}\right)-\mathscr{F}\left(F_{3} \otimes F_{2}\right)+\mathscr{F}\left(D_{2}\left(F_{3}\right)\right)+\mathscr{F}\left(S_{2}(W)^{\wedge}\right) .
$$

By considering several cases, we shall see that this is unlikely to be the Hilbert series of a positively graded free module. Denote the degrees of the generators of $F_{1}$, $F_{2}$, and $F_{3}$ by $\alpha=d, \beta=d+a$, and $\gamma=d+a+b$ respectively.

(4.2) IDEALS OF TYPE TWO. From Proposition 2.8 we have the exact sequence

$$
0 \rightarrow S_{2}(W)^{\wedge} \rightarrow S^{2}(-\gamma) \rightarrow W(-2 \gamma) \rightarrow 0 .
$$

(4.2.1) Solving for $\mathscr{F}\left(S_{2}(W)^{\wedge}\right)$ and substituting into $\mathscr{F}(K)$ we obtain: $\mathscr{F}(K)=$ $\mathscr{F}\left(\Lambda^{2} F_{2}\right)+\mathscr{F}\left(F_{2}{ }^{*}(-2 \gamma)\right)-\mathscr{F}\left(F_{1}{ }^{*}(-2 \gamma)\right)$. This implies that $2 \gamma-\alpha$ be equal to $2 \gamma-\beta$ or to $2 \beta$. The first is clearly impossible, while by the formula of [HK], the type of $S$ is given by

$$
p=\alpha \cdot \beta /(\gamma-\alpha)(\gamma-\beta)
$$


For $p=2$ we obtain that $\beta=\gamma-\alpha$. But an ideal with a pure resolution and this numerical data is Gorenstein [KMU, Proposition 2.4].

(4.2.2) We can describe the numerical data for a resolution of $\mathbf{Z}_{2}$, and as a consequence show conditions for the vanishing of the deformation module $T_{3}(S / R, S)$.

Because $H_{1}$ is Cohen-Macaulay, the module $\mathbf{Z}_{2}$ has projective dimension 1; we compute its Hilbert series. From (2.1) we have the exact sequence

$$
0 \rightarrow \Lambda^{2} \mathbf{Z}_{1} \rightarrow \mathbf{Z}_{2} \rightarrow D \rightarrow 0
$$

where $D=\operatorname{Ext}^{3}\left(S_{2}(W), R\right)$. On the other hand, $S_{2}(W)$ is given as above by Proposition 2.8.

We can therefore put together the Hilbert series of $\mathbf{Z}_{2}$ (we only write the numerators):

$$
\mathscr{F}\left(\mathbf{Z}_{2}\right)=\left[\begin{array}{c}
b_{2} \\
2
\end{array}\right] \lambda^{2 \beta}+b_{2} \lambda^{2 \gamma-\beta}-2 b_{1} \lambda^{\alpha+\gamma}-b_{1} \lambda^{2 \gamma-\alpha} .
$$

Since $\operatorname{pd} \mathbf{Z}_{2}=1$, we easily get the Betti numbers and degrees of a resolution of $\mathbf{Z}_{2}$.

(4.2.3) Let us give one application. The deformation module $T_{3}(S / R, S)$ may be described as the quotient $H_{2} / H_{1}^{2}$ [He $\mathbf{H e}_{3}$. Its vanishing means therefore that $\mathbf{Z}_{2}=\mathbf{Z}_{1}^{2}$ $+B_{2}$. Since $B_{2}$ is generated by elements of degree $3 \alpha$, we must have $3 \alpha \geqslant 2 \gamma-\beta$, which is a contradiction by [KMU, Proposition 2.4].

(4.2.4) This resolution will now be used to show that although ideals of type 2 lie in the linkage class of ideals of deviation two (that are therefore SCM), the $\mathrm{H}_{2}$ are not necessarily Cohen-Macaulay. A case in point is Example 2.6 of [KMU]. It is a radical ideal of height three in a polynomial ring of dimension four with a resolution (with $a=1$ ):

$$
0 \rightarrow R^{2}(-6 a) \rightarrow R^{9}(-4 a) \rightarrow R^{8}(-3 a) \rightarrow R \rightarrow S \rightarrow 0 .
$$

To show that a generic complete intersection with this numerical data cannot have $H_{2}(I)$ Cohen-Macaulay, we proceed as follows. First, since $H_{1}(I)$ is CohenMacaulay - as its type is two-we may specialize down to a polynomial ring in three variables. We compare the expected length of $H_{2}(I)\left(\mathrm{cf}\right.$. $\left.\left[\mathbf{H e}_{1}\right]\right)$ with values derived from its Hilbert function.

Because the rank of $\mathrm{H}_{2}(I)$-prior to the specialization-is 10 , and the multiplicity of $S$-derived from the resolution-is $12 a^{3}$, we are going to show that $\sum_{i=0}^{x} l\left(H_{2}(I)_{i}\right)-120 a^{3}$ is strictly positive for an appropriate $x$. It suffices to carry out the following sequence of computations and substitutions, where we replace the Hilbert function of $B_{2}$ in terms of that of $K_{3}$ and $\mathbf{Z}_{3}$.

$$
\begin{aligned}
\sum_{i=0}^{x} l\left(H_{2}\right)_{i}-120 a^{3}=\sum_{i=0}^{x} l\left(\mathbf{Z}_{2}\right)_{i}-\sum_{i=0}^{x} l\left(B_{2}\right)_{i} \\
=36\left[\begin{array}{c}
x-8 a+3 \\
3
\end{array}\right]+9\left[\begin{array}{c}
x-8 a+3 \\
3
\end{array}\right]-16\left[\begin{array}{c}
x-9 a+3 \\
3
\end{array}\right] \\
\quad-8\left[\begin{array}{c}
x-9 a+3 \\
3
\end{array}\right]-56\left[\begin{array}{c}
x-9 a+3 \\
3
\end{array}\right]-120 a^{3}+\sum_{i=0}^{x} l\left(\mathbf{Z}_{3}\right)_{i} .
\end{aligned}
$$


For $x=12 a-2$ this expression evaluates to $72 a^{2}+2 a+\left(\right.$ contribution of $\mathbf{Z}_{3}$ ). Therefore $\mathrm{H}_{2}(I)$ is not Cohen-Macaulay.

(4.3) IDEALS OF DEVIATION Two. We shall look at the question of the conormal module for these ideals. Again we have an explicit form for a resolution of $\mathbf{Z}_{2}$.

(4.4) Proposition. Let I be a Cohen-Macaulay ideal of height three generated by the homogeneous elements $\left\{f_{1}, \ldots, f_{n}\right\}$. Let $d_{i}=\operatorname{degree}\left(f_{i}\right)$ and set $e=\sum d_{i}$. Then the projective resolution of $\mathbf{Z}_{n-3}$ is:

$$
0 \rightarrow F_{2}^{*}(-e) \rightarrow F_{3}^{*}(-e) \oplus \Lambda^{n-2} F_{1} \rightarrow \mathbf{Z}_{n-3} \rightarrow 0 .
$$

Proof. We already know that $\mathbf{Z}_{n-3}$ has projective dimension 1 (1.3.2). We get its numerical data from the two exact sequences

$$
\begin{aligned}
& 0 \rightarrow K_{n} \rightarrow K_{n-1} \rightarrow K_{n-2} \rightarrow B_{n-3} \rightarrow 0, \\
& 0 \rightarrow B_{n-3} \rightarrow \mathbf{Z}_{n-3} \rightarrow H_{n-3} \rightarrow 0 .
\end{aligned}
$$

What is needed is the precise relationship-in the graded case-between the canonical module $W$ and $H_{n-3}$. By keeping track of the degrees in the usual identification, J. Herzog pointed out the following (valid in all codimensions).

(4.5) LEMMA. $H_{n-3} \cong W(-e)$.

To proceed with the proof of (4.4) we have successively

$$
\begin{aligned}
\mathscr{F}\left(Z_{n-3}\right)= & \mathscr{F}\left(H_{n-3}\right)+\mathscr{F}\left(B_{n-3}\right) \\
= & \mathscr{F}\left(K_{n-2}\right)-\mathscr{F}\left(K_{n-1}\right)+\mathscr{F}\left(K_{n}\right)+\mathscr{F}\left(F_{3}{ }^{*}(-e)\right) \\
& -\mathscr{F}\left(F_{2}{ }^{*}(-e)\right)+\mathscr{F}\left(F_{1}{ }^{*}(-e)\right)-\mathscr{F}(R(-e)) .
\end{aligned}
$$

Noting that $R(-e)=K_{n}$ and $F_{0}^{*}(-e)=K_{n-1}$ we obtain

$$
\mathscr{F}\left(\mathbf{Z}_{n-3}\right)=\mathscr{F}\left(F_{2}^{*}(-e)\right)+\mathscr{F}\left(K_{n-2}\right)-\mathscr{F}\left(F_{1}^{*}(-e)\right) .
$$

Taking into account that $\mathbf{Z}_{n-3}$ has projective dimension one and can be generated by $v\left(F_{2}\right)+v\left(K_{n-2}\right)$ elements, it follows that the resolution of $\mathbf{Z}_{n-3}$ is as asserted.

We can now show that for ideals of deviation two, with pure resolutions, $S_{2}(I)$ cannot have projective dimension two. Indeed, the Hilbert series of $K$ is (again only numerators are shown)

$$
\begin{aligned}
\mathscr{F}(K)= & b_{2} b_{3} \lambda^{2 d+2 a+b}-\left[\begin{array}{c}
b_{2}+1 \\
2
\end{array}\right] \lambda^{2(d+a)}+15 \lambda^{2 d}-5 b_{2} \lambda^{d+a}+5 b_{3} \lambda^{d+a+b} \\
& +b_{3} \lambda^{4 d-a-b}-b_{2} \lambda^{4 d-a}+10 \lambda^{3 d} .
\end{aligned}
$$

As it may be assumed that $d \geqslant a+b$ (cf. [KMU, Proposition 2.4]), the term of degree $d+a$ has no match among the terms with positive coefficients. It follows that $K$ cannot be a free module.

5. Computational notes. We discuss, in broad outline, how to decide if an ideal of a polynomial ring, given by a set of generators, is syzygetic or of linear type, and then whether some of its Koszul homology modules are Cohen-Macaulay. 
There exists a simulacrum of a division algorithm for the ring of polynomials in several variables. Set $R=k\left[x_{1}, \ldots, x_{n}\right]$ and identify the set of monomials of $R$ with the additive semigroup $\mathbf{N}^{n}$. Pick a well ordering for $\mathbf{N}^{n}$ which is compatible with its semigroup structure. Finally, define the 'degree' of a monomial as the corresponding vector of exponents in $\mathbf{N}^{n}$, and the degree $d(f)$ of a nonzero polynomial $f$ of $R$ as the supremum of the degrees of the monomials that occur with nonzero coefficients in $f$.

For an ideal $I$, define $d(I)$ as the union of the degrees of the nonzero elements of I. $d(I)$ is a subsemigroup of $\mathbf{N}^{n}$, satisfying $d(I)+\mathbf{N}^{n} \subset d(I)$. By the Hilbert basis theorem it follows that

$$
d(I)=\bigcup\left(d\left(f_{i}\right)+\mathbf{N}^{n}\right), \quad 1 \leqslant i \leqslant r .
$$

One can then verify that the images of the monomials $g, d(g) \notin d(I)$, form a vector space basis for $R / I$ over $k$.

(5.1) Definition. $\left(f_{1}, \ldots, f_{r}\right)$ is a Gröbner basis of $I$.

Partly, the detailed study of this basis goes back to Macaulay. Presently there exist several implementations of an algorithm of Buchberger that constructs Gröbner bases. $[\mathrm{Bu}]$ is a beautiful exposition of the algorithm and its capabilities.

Some of its properties are:

(5.1i) A Gröbner basis of $I$ is a generating set for the ideal and may be readily used to test membership in $I$. Indeed, the condition on the $\left(f_{i}\right)$ is equivalent to: Every element $f$ of $I$ can be written as $f=\sum a_{i} f_{i}$, with $d(f) \geqslant d\left(a_{i} f_{i}\right)$.

(5.1ii) Given an ideal $I$ of $k\left[x_{1}, \ldots, x_{n}\right]$, find the contraction of $I$ to the subring of $R$ defined by a subset of the indeterminates. It suffices to find a Gröbner basis of $I$ corresponding to an ordering of the variables that lists the variables of the subring first. The desired intersection is generated by the basis elements contained in the subring.

In turn this property may be used to compute ( $T$ is an indeterminate over $R$ ):

(5.2) The intersection of two ideals $A$ and $B: A \cap B=(A \cdot T, B(T-1)) \cap R$.

(5.3) Decide whether an element $f$ of $R$ is regular modulo an ideal $A$ : that is, compute $A: f(=(A \cdot T,(1-T) f) \cap R) / f)$ and compare it to $A$. Similarly one can compute $\bigcup_{n \geqslant 1} A: f^{n}$ as $(A \cdot T,(1-T f)) \cap R=(A,(1-T f)) \cap R$. In fact, because of the last equality, we have found determining the ideal $\bigcup_{n \geqslant 1} A: f^{n}$ to be a convenient procedure to compute depths of cyclic modules.

(5.4) Noether normalizaton, height of an ideal, and systems of parameters of affine algebras. Given a nonzero ideal $P=\left(h_{1}, \ldots, h_{s}\right)$ of the polynomial ring $B=Q\left[x_{1}, \ldots, x_{n}\right]$, a Noether normalization of the affine algebra $B / P$ can be obtained as follows. Effect a linear change of variables-done on-line, usually-so that one of the elements of $P, f_{1}$, is monic in one of the variables, say $x_{n}$. Consider $P_{1}=P \cap B_{1}, B_{1}=Q\left[x_{1}, \ldots, x_{n-1}\right]$. Note that $B / P$ is integral over $B_{1} / P_{1}$, so that in particular $\mathrm{ht}(P)=\mathrm{ht}\left(P_{1}\right)+1$. In this manner heights, analytic spreads, and systems of parameters can be computed. When all the changes of variables are taken into account we have a sequence of elements $f_{i}\left(y_{1}, \ldots, y_{n-i+1}\right) \in B=Q\left[y_{1}, \ldots, y_{n}\right]$, $1 \leqslant i \leqslant \operatorname{height}(I)$, monic in $y_{n-i+1}$, lying in the ideal $I$. 
(5.5) To find whether the ideal $I$ is syzygetic or of linear type, we first determine a presentation of the Rees algebra of $I$. If $I$ is generated by $\left\{f_{1}, \ldots, f_{m}\right\}$, the kernel of the homomorphism

$$
B=R\left[T_{1}, \ldots, T_{m}\right] \stackrel{\phi}{\rightarrow} \mathscr{R}(I), \quad \phi\left(T_{i}\right)=f_{i} t,
$$

is simply the intersection of $B$ with the ideal of $B[T]$ generated by $\left\{T_{1}-f_{1} T, \ldots, T_{m}\right.$ $\left.-f_{m} T\right\}$ (cf. [BSV]). We may then test for $J_{2}=J_{1} \cdot B_{1}$ or $J=J_{1} \cdot B$, according to (1.1.3).

We now indicate how to determine whether $H_{1}(I)$ is Cohen-Macaulay. More precisely, we shall test for that condition at a maximal ideal. It works best, therefore, if $I$ is a homogeneous ideal.

(5.6) Let I be a homogeneous, Cohen-Macaulay, syzygetic ideal of height $g$. Let $k\left[y_{1}, \ldots, y_{n-g}\right]$ be a Noether normalization of $k\left[y_{1}, \ldots, y_{n}\right] / I$. Then $H_{1}(I)$ is CohenMacaulay if and only if the following condition holds:

(\#) $I^{2} \cap\left(\left(y_{1}, \ldots, y_{i-1}\right) I^{2}: y_{i}\right)=\left(y_{1}, \ldots, y_{i-1}\right) I^{2}$ for $i=1, \ldots, n-g$.

Proof. Because of the exact sequence

$$
0 \rightarrow H_{1}(I) \rightarrow S^{r} \rightarrow I / I^{2} \rightarrow 0
$$

we must show that $\operatorname{depth}\left(I / I^{2}\right) \geqslant n-g-1$. Since depth $I=n-g+1$, it suffices to show that $\operatorname{depth}\left(R / I^{2}\right) \geqslant n-g-1$ using any system of parameters for $R / I^{2}$. We therefore make use of the images of the $y_{i}, 1 \leqslant i \leqslant n-g$, which translates into the condition above.

This method allowed Villarreal [Vi] to discover some classes of SCM ideals generated by monomials.

(5.7) We are simply verifying whether $\left\{y_{1}, \ldots, y_{n-g}\right\}$ is a regular sequence on $I^{2}$. This could also be effected in the following direct manner. Let $T_{1}, \ldots, T_{n-g}$ be indeterminates and denote by $\mathbf{Z}$ the module of cycles of the regular sequence $\mathbf{y}=\left\{y_{1}, \ldots, y_{n-g}\right\}$. Then $\mathbf{y}$ is a regular sequence on $I^{2}$ if and only if

$$
\mathbf{Z} \cap I^{2} \cdot\left(T_{1}, \ldots, T_{n-g}\right)=I^{2} \cdot \mathbf{Z} .
$$

Furthermore, if $I$ is no longer a homogeneous ideal, we can still use this approach to test for Cohen-Macaulayness at any maximal ideal lying over (y).

(5.8) A more direct method, not requiring that $I$ be syzygetic, but assuming that $I$ is generically a complete intersection, would simply compute the dimension of the vector space $\mathbf{Z}_{1}(I) /\left(\mathbf{y} \mathbf{Z}_{1}(I)+B_{1}(I)\right)$, where now $\mathbf{Z}_{1}(I)$ and $B_{1}(I)$ are the 1-cycles and boundaries of a generating set of $I$. This length would be compared to the expected length of this module (cf. [He 1 ).

If $I$ is not syzygetic, we may still obtain information about its Koszul homology. Suppose $I$ is a complete intersection in codimension at most one-e.g., suppose $R / I$ is normal. Let us show how to test for the Cohen-Macaulayness of the 'dual' of $H_{1}(I)$, that is, of $H_{m-g-1}(I)$. 
It is easy to see that dualizing the exact sequence

$$
0 \rightarrow \delta(I) \rightarrow H_{1}(I) \rightarrow S^{m} \rightarrow I / I^{2} \rightarrow 0
$$

twice with respect to the canonical module $W$, and using the hypothesis on $I$, we obtain the exact sequence

$$
0 \rightarrow \operatorname{Hom}_{S}\left(H_{m-g-1}(I), W\right) \rightarrow S^{m} \rightarrow I / I^{(2)} \rightarrow 0 .
$$

Here $I^{(2)}$ is the symbolic square of $I$, that is, the intersection of the primary components of $I^{2}$ corresponding to its minimal primes. If $f$ is an element regular on $R / I$ such that $I_{f}$ is complete intersection, then $I^{(2)}=I_{f}^{(2)} \cap R$. Before we describe how $f$ and $I^{(2)}$ are determined, note the following analog of the previous proposition.

(5.9) Let $I$ be a Cohen-Macaulay ideal that is a complete intersection in codimension 1. Then $H_{r-g-1}(I)$ is Cohen-Macaulay at the ideal $\left(y_{1}, \ldots, y_{n}\right) / I$ if and only if

$(\# \#) \quad I^{(2)} \cap\left(\left(y_{1}, \ldots, y_{i-1}\right) I^{(2)}: y_{i}\right)=\left(y_{1}, \ldots, y_{i-1}\right) I^{(2)}$ for $i=2, \ldots, n-g$.

Assuming that $f$ has been found, $I^{(2)}$ is simply the ideal $\bigcup_{s \geqslant 1}\left(I^{2}: f^{s}\right)$. According to $(5.8) I^{(2)}$ is computed as follows:

$$
I^{(2)}=\left(I^{2}, 1-T f\right) \cap R .
$$

Depending on the origin of $I$, there are faster ways of finding $f$ (e.g., Jacobian ideal). Here is a crude but general method. Let

$$
R^{r} \stackrel{\phi}{\rightarrow} R^{m} \rightarrow I \rightarrow 0
$$

be a presentation of $I$. To say that $I$ is a complete intersection in codimenion 1 means that the ideal $J$ generated by the minors of $\phi$ is not contained in any ideal of height $g+1$ that contains $I$; it follows that this part of the hypothesis can also be tested for. While the Noether normalization $R / I$ is being carried out we can also keep track of the successive intersections of $I+J$; any element left at the end of normalization would serve the purpose. For convenience purposes 'shorter' subideals of $J$ should be tried first.

(5.10) EXAMPLE. Let $I$ be the ideal generated by the $2 \times 2$ minors of the generic matrix:

$$
\left[\begin{array}{lll}
x_{11} & x_{12} & x_{13} \\
x_{21} & x_{22} & x_{23} \\
x_{31} & x_{32} & x_{33}
\end{array}\right] .
$$

It was verified that $I$ is of linear type. In fact, Huneke $\left[\mathbf{H u}_{3}\right]$ showed that the ideal of submaximal minors of a generic square matrix is of linear type for all dimensions.

Letting the variable $T_{i j}$ correspond to the $(i, j)$-minor in a presentation of the Rees algebra of $I$ we obtained the following system of parameters for the Rees algebra.

$$
\begin{aligned}
& \left\{x_{33}, T_{11}, T_{21}-x_{32}, T_{22}-x_{23}, T_{12}-x_{11},\right. \\
& \left.\quad T_{31}-x_{12}, T_{33}-x_{13}, T_{32}-x_{21}, T_{23}-x_{22}, T_{13}-x_{31}\right\} .
\end{aligned}
$$


Furthermore it was shown that the Rees algebra is Cohen-Macaulay and, using the criterion of [BSV], normal. Both of these facts were not known. In contrast, as indicated in Theorem 3.7, $I$ cannot be generated by a $d$-sequence.

There are other approaches to these computations. As tried in the Macsyma system it was very voracious of CPU time (several hours in a DEC-20). The system of parameters was found by 'hand' rather than computed. It was fairly cheap to find that the Rees algebra modulo the maximal ideal of the normalizing subring $C$ has dimension 40 over $Q$. For an alternative proof, all that is needed is a proof that the rank of $R(I)$ over $C$ is also 40 .

(5.11) ExAmple. Let $I$ be a homogeneous Cohen-Macaulay ideal of codimension 3 or 4 , which is generically a complete intersection. After a system of parameters (of degree 1) for $R / I$ has been found, testing whether $I / I^{2}$ is Cohen-Macaulay is easy: As it involves 3 or 4 variables, finding Gröbner bases for the 0 -dimensional ideals $I$ and $I^{2}$ - and then the vector space dimensions of $R / I$ and $R / I^{2}-$ is inexpensive. This method was used in the examples of (3.3).

\section{BIBLIOGRAPHY}

[A] R. Apery, Sur les courbes de premiere espece de l'espace à trois dimensions, C. R. Acad. Sci. Paris 220 (1945), 271-272.

[AB] M. Auslander and M. Bridger, Stable module theory, Mem. Amer. Math. Soc. No. 94, 1969.

[AH] L. Avramov and J. Herzog, The Koszul algebra of a codimension 2 embedding, Math. Z. 175 (1980), 249-260.

[BSV] P. Brumatti, A. Simis and W. V. Vasconcelos, Normal Rees algebras, J. Algebra (to appear).

[Bu] B. Buchberger, An algorithmic method in polynomial ideal theory, Recent Trends in Mathematical Systems Theory (N. K. Bose, ed.), Reidel, Dordrecht, 1985.

[BE] D. Buchsbaum and D. Eisenbud, Algebra structures for finite free resolutions, and some structure theorems for ideals of codimension 3, Amer. J. Math. 99 (1977), 447-485.

[BU] R.-O. Buchweitz and B. Ulrich, Homological properties which are invriant under linkage, preprint, 1983.

[Ga] F. Gaeta, Quelques progrès récents dans la classification des variètés algébriques d'un espace projective, Deuxième Colloque de Géométrie Algébrique, Thone, Liège, 1952, pp. 145-183.

[Go] S. Goto, The divisor class group of a certain Krull domain, J. Math. Kyoto Univ. 17 (1977), 47-50.

[HO] R. Hartshorne and A. Ogus, On the factorlality of local rings of small embedding codimension, Comm. Algebra 1 (1974), 415-437.

$\left[\mathbf{H e}_{1}\right] \mathrm{J}$. Herzog, Ein Cohen-Macaulay-Kriterium mit Anwendungen auf den Konormalenmodul und den Differentialmodul, Math. Z. 163 (1978), 149-162.

$\left[\mathrm{He}_{2}\right] \ldots$, Komplexe, Auflösungen und Dualität in der lokalen Algebra, Habilitationsschrift, Regensburg Universität, 1974.

$\left[\mathrm{He}_{3}\right]$, Homological properties of the module of differentials, Atas VI Escola de Algebra, Soc. Brasileira de Matematica, 1981, pp. 33-64.

[HK] J. Herzog and M. Kühl, On the Betti numbers of finite pure and linear resolutions, Comm. Algebra 12 (1984), 1627-1646.

[HM] J. Herzog and M. Miller, Gorenstein ideals of deviation two, Comm. Algebra 13 (1985), $1977-1990$.

$\left[\right.$ HSV $\left._{1}\right]$ J. Herzog, A. Simis and W. V. Vasconcelos, Koszul homology and blowing up rings, Proc. Commutative Algebra Trento Conf., Lecture Notes in Pure and Applied Math., vol. 84, Marcel Dekker, New York, 1983, pp. 79-169.

$\left[\mathbf{H S V}_{2}\right] \ldots$ On the arithmetic and homology of algebras of linear type, Trans. Amer. Math. Soc. 283 (1984), 661-683.

[HVV] J. Herzog, W. V. Vasconcelos and R. Villarreal, ldeals with sliding depth, Nagoya Math. J. 99 (1985), 159-172.

$\left[\mathbf{H u}_{\mathbf{1}}\right]$ C. Huneke, Linkage and the Koszul homology of ideals, Amer. J. Math. 104 (1982), 1043-1062. 
$\left[\mathrm{Hu}_{2}\right] \ldots$ Strongly Cohen-Macaulay schemes and residual intersections, Trans. Amer. Math. Soc. 277 (1983), 739-763.

$\left[\mathrm{Hu}_{3}\right] \ldots$, Determinantal ideals of linear type, Arch. Math. 47 (1986), 324-329.

[K] E. Kunz, Almost complete intersections are not Gorenstein rings, J. Algebra 28 (1974), 111-115.

[KMU] A. R. Kustin, M. Miller and B. Ulrich, Linkage theory for algebras with pure resolutions, J. Algebra 102 (1986), 199-228.

[Le] K. Lebelt, Freie Auflösungen äußeser Potenzen, Manuscripta Math. 21 (1977), 341-355.

[PS] C. Peskine and L. Szpiro, Liaison des variétés algébriques, Invent. Math. 26 (1974), 271-302.

[SV] A. Simis and W. V. Vasconcelos, The syzygies of the conormal module, Amer. J. Math. 103 (1981), $203-224$.

[V] W. V. Vasconcelos, Ideals generated by R-sequences, J. Algebra 6 (1967), 309-316.

[VV] W. V. Vasconcelos and R. Villarreal, On Gorenstein ideals of codimension four, Proc. Amer. Math. Soc. 98 (1986), 205-210.

[Vi] R. Villarreal, Koszul homologv of Cohen-Macaulay ideals, Ph. D. Thesis, Rutgers University, 1986.

[Wa] J. Watanabe, A note on Gorenstein rings of embedding codimension 3, Nagoya Math. J. 50 (1973), $227-232$.

[We] J. Weyman, Resolutions of the exterior and symmetric powers of a module, J. Algebra 58 (1979), $333-341$.

Department of Mathematics, Rutgers University, New Brunswick, New Jersey 08903 